\title{
A ELETROMIOGRAFIA DE SUPERFÍCIE NOS PERIÓDICOS NACIONAIS EM FONOAUDIOLOGIA
}

\section{Surface electromyography in national journals in speech, language and hearing sciences}

\author{
Leandro de Araújo Pernambuco (1), Renata Andrade da Cunha ${ }^{(2)}$, Otávio Lins ${ }^{(3)}$, \\ Jair Carneiro Leão ${ }^{(4)}$, Hilton Justino da Silva ${ }^{(5)}$
}

\begin{abstract}
RESUMO
Tema: eletromiografia de superfície nos periódicos nacionais em Fonoaudiologia. Objetivo: caracterizar a abordagem da eletromiografia de superfície nos periódicos nacionais em Fonoaudiologia. Conclusão: poucos estudos abordam a eletromiografia de superfície nos periódicos nacionais em Fonoaudiologia, com maior concentração nas áreas de motricidade orofacial e fluência revelando uma maior necessidade de aprofundar esse assunto e diversificar os temas de interesse fonoaudiológico.
\end{abstract}

DESCRITORES: Eletromiografia; Publicações Periódicas; Publicações Científicas e Técnicas

\section{INTRODUÇÃO}

A eletromiografia de superfície (EMGs) é o método de registro das variações da atividade elétrica muscular durante sua contração ${ }^{1-3}$. Avalia principalmente as condições fisiológicas e patológicas do músculo, fornece informações sobre os princípios que regem a função muscular ${ }^{1}$ e pode

(1) Fonoaudiólogo do Hospital do Câncer de Pernambuco, HCP, Recife, PE, Brasil; Prefeitura Municipal do Jaboatão dos Guararapes, Jaboatão dos Guararapes, PE, Brasil; Mestrando em Ciências da Saúde na Universidade Federal de Pernambuco.

(2) Fisioterapeuta; Especializanda em Fisioterapia Neurofuncional na Faculdade Integrada do Recife.

(3) Médico Neurologista; Professor Adjunto do Departamento de Neuropsiquiatria da Universidade Federal de Pernambuco, UFPE, Recife, PE, Brasil; Mestre em Fisiologia pela Universidade de Otawa, Canadá; Doutor em Medicina Neurológica pela Universidade Federal de São Paulo.

(4) Cirurgião Dentista; Professor Associado I do Departamento de Clínica e Odontologia Preventiva da Universidade Federal de Pernambuco, UFPE, Recife, PE, Brasil; Pós-doutorado pela Universidade de Londres, Inglaterra.

(5) Fonoaudiólogo; Professor Adjunto I do curso de Fonoaudiologia da Universidade Federal de Pernambuco, UFPE, Recife, PE, Brasil; Vice-coordenador da Pós-graduação em Patologia da Universidade Federal de Pernambuco, Recife, PE, Brasil; Doutor em Nutrição pela Universidade Federal de Pernambuco.

Conflito de interesses: inexistente contribuir com informações importantes para o diagnóstico ${ }^{4}$.

A EMGs tem sido considerada o instrumento mais preciso para avaliar a atividade elétrica dos músculos orofaciais, devido à facilidade em relação a outros parâmetros de mensuração. Caracterizase por ser não-invasivo, livre de desconforto e radiação, rápido, barato e de fácil compreensão pelo paciente ${ }^{5,6}$. Vem sendo amplamente utilizada durante reabilitação muscular e funcional, servindo como instrumento quantificador da atividade muscular ${ }^{7}$, podendo auxiliar no diagnóstico e terapêutica dos distúrbios motores orofaciais, como a mastigação e a deglutição ${ }^{3}$, oferecendo a possibilidade de analisar a ação de um grupo muscular ou de um feixe muscular específico ${ }^{2}$.

O registro eletromiográfico requer um sistema que compreende os eletrodos que capturam os potenciais elétricos (atividade) do músculo em contração (fase de entrada da informação); um amplificador, que processa o pequeno sinal elétrico (fase de processamento); um decodificador, que permite a visualização gráfica e/ou audição dos sons, o que permitirá a completa análise dos dados (fase de saída de informação) ${ }^{8}$.

A instrumentação eletromiográfica está relacionada com quatro itens distintos: a origem do sinal, o transdutor utilizado na detecção do sinal (eletrodo), o amplificador do sinal elétrico e o circuito de processamento do sinal ${ }^{9}$. 
Como qualquer outro método, a aplicabilidade da eletromiografia apresenta algumas limitações. Os sinais eletromiográficos podem ser afetados pela anatomia e propriedades fisiológicas dos músculos, pelo controle do sistema nervoso periférico, pela instrumentação usada na coleta dos sinais ${ }^{10}$, presença de má oclusões, interferências oclusais, treino muscular, tipologia facial e alimentação ${ }^{11}$. A espessura e camada de gordura na pele, posicionamento dos eletrodos e a motivação do paciente em realizar o exame também podem influenciar nos resultados ${ }^{12}$. Além disso, as diferenças interindividuais dificultam a determinação de diferenças quantitativas significantes entre os indivíduos neste tipo de exame ${ }^{13}$.

$\mathrm{Na}$ clínica fonoaudiológica, a eletromiografia é considerada um importante auxiliar no entendimento dos padrões de atividade elétrica dos músculos faciais e mastigatórios, contribuindo com um diagnóstico mais objetivo e intervenção mais efetiva ${ }^{14}$. Ao contrário de avaliações mais subjetivas dos grupos musculares, tais como a palpação ou inspeção visual, o caráter mais objetivo da eletromiografia pode complementar os dados do diagnóstico, tratamento e até mesmo, prognósticos dos casos na clínica fonoaudiológica ${ }^{15}$.

A variabilidade da aplicação da EMGs na Fonoaudiologia pode ser endossada pelos diversos estudos nesta área, envolvendo, por exemplo, indivíduos respiradores orais ${ }^{14}$, com paralisia facial ${ }^{16}$, disfunções têmporo-mandibulares ${ }^{10}$, alterações vestibulares ${ }^{17}$, usuários de prótese dentária ${ }^{18}$, dentre outros.

Estudos de natureza cartográfica, que realizam um balanço da construção do conhecimento, vêm sendo realizados por serem considerados importantes no processo de construção da memória e do aperfeiçoamento do conhecimento ${ }^{19}$. Por ser uma área ainda recente na Fonoaudiologia, percebe-se que poucos trabalhos foram realizados, daí a importância de conhecer qual o espaço que este tema já possui nos periódicos nacionais especializados nesta ciência e, desta forma, compreender o que ainda necessita ser explorado.

O objetivo desta pesquisa foi caracterizar os periódicos nacionais em Fonoaudiologia em relação à abordagem sobre eletromiografia de superfície nos artigos publicados de janeiro de 2000 a dezembro de 2008.

\section{MÉTODOS}

Para este estudo, de natureza cartográfica, foi realizada busca na base de dados LILACS - Literatura Latino Americana e do Caribe em Ciências da Saúde, que comporta cinco periódicos nacionais especializados em Fonoaudiologia: Revista CEFAC, Distúrbios da Comunicação, Revista Pró-Fono, Revista da Sociedade Brasileira de Fonoaudiologia e Fono Atual. Todas são registradas no Instituto Brasileiro de Ciências e Tecnologia (IBICT) e, portanto, possuidoras do International Standard Serial Number (ISSN). Todos os periódicos analisados estavam classificados no Qualis como maior ou igual à nacional B. O Qualis, concebido pela Coordenadoria de Aperfeiçoamento de Pessoal de Nível Superior (CAPES), é o resultado da classificação dos veículos utilizados pelos Programas de Pós-Graduação para a divulgação de sua produção intelectual ${ }^{20}$. Foram analisados todos os artigos publicados entre janeiro de 2000 e dezembro de 2008, considerando como critério para busca o termo livre "eletromiografia".

As variáveis consideradas para análise foram: número de publicações por periódico, por ano, por tipo de artigo, por área e por tema. Os dados foram analisados de forma descritiva com auxílio de tabelas e gráficos.

Não foram citados nomes dos autores dos artigos e as revistas científicas por considerações éticas e os periódicos foram nomeados pelas letras $A, B, C, D$ e E através de um sorteio ${ }^{21}$.

\section{REVISÃO DE LITERATURA}

$\mathrm{Na}$ Tabela 1 encontra-se a caracterização dos periódicos em relação ao período de indexação na base de dados LILACS. Também na Tabela 1 é possível observar o número total de artigos publicados por cada periódico no período estudado, bem como o número de artigos relacionados a eletromiografia de superfície.

A Tabela 1 permite também verificar que a proporção de artigos sobre eletromiografia de superfície nestes periódicos é muito pequena. Dos 954 artigos publicados no período de 2000 a 2008, 10 estão relacionados a este tema, o que representa cerca de $1 \%$ do total. Nesta mesma tabela, observa-se que existe uma maior concentração de pesquisas publicadas no periódico $\mathrm{C}$, tanto em relação ao total de artigos, quanto àqueles sobre EMGs. Este periódico também é o único que esteve indexado durante todo o período considerado nesta análise e é também o que apresenta melhor classificação no Qualis.

A Figura 1 mostra a distribuição das publicações ao longo do tempo. Percebe-se que a primeira publicação surge apenas dois anos após o início do período de análise e que depois ocorre uma estabilidade com pelo menos uma publicação a cada ano, até o ano de 2008, quando o número de publicações aumentou para 4. 
Tabela 1 - Classificação dos periódicos nacionais em fonoaudiologia, segundo início e final do período de indexação na base de dados LILACS, número total de artigos publicados no período de estudo, número de artigos sobre eletromiografia publicados no período estudado

\begin{tabular}{|c|c|c|c|c|}
\hline Revista & Início & Final & $\begin{array}{c}\text { № artigos } \\
\text { jan/2000-dez/2008 }\end{array}$ & $\begin{array}{l}\text { № artigos EMGs } \\
\text { jan/2000-dez/2008 }\end{array}$ \\
\hline A & $\begin{array}{c}\text { v.8, n.2 } \\
2006\end{array}$ & $\begin{array}{l}\text { Até os dias } \\
\text { atuais }\end{array}$ & 177 & 1 \\
\hline B & $\begin{array}{c}\text { v.8, n.1 } \\
1996\end{array}$ & $\begin{array}{l}\text { v.19, n.3 } \\
2007\end{array}$ & 182 & 1 \\
\hline C & $\begin{array}{c}\text { v. } 1, \mathrm{n} .1 \\
1989\end{array}$ & $\begin{array}{l}\text { Até os dias } \\
\text { atuais }\end{array}$ & 340 & 7 \\
\hline$D$ & $\begin{array}{c}\text { v.11, n.1 } \\
2006\end{array}$ & $\begin{array}{l}\text { Até os dias } \\
\text { atuais }\end{array}$ & 147 & 1 \\
\hline$E$ & $\begin{array}{c}\text { v. } 4, \text { n. } 17 \\
2001\end{array}$ & $\begin{array}{c}\text { v.8, n.35 } \\
2006\end{array}$ & 108 & 0 \\
\hline \multicolumn{3}{|c|}{ TOTAL } & 954 & 10 \\
\hline
\end{tabular}

* Considerados os artigos publicados apenas durante o período de indexação de cada periódico.

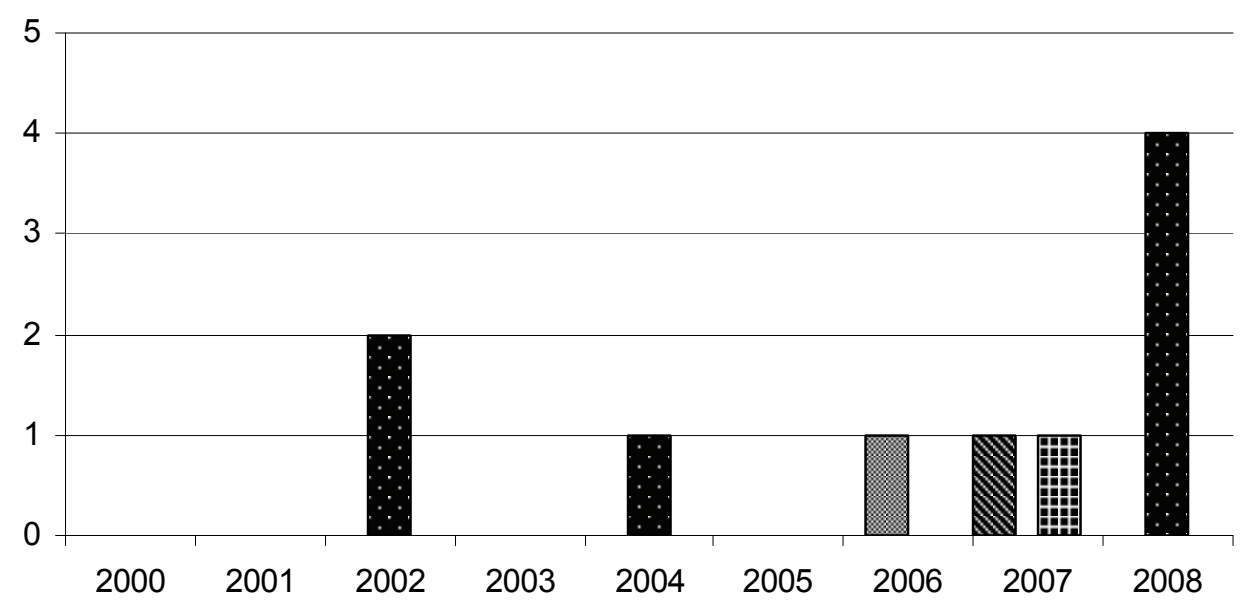

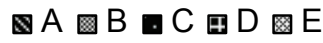

Figura 1 - Distribuição das publicações sobre EMGs nos periódicos nacionais em Fonoaudiologia, segundo $o$ ano

A Figura 2 relaciona-se ao tipo de artigo aplicado nas publicações. Constatou-se que todos os 10 artigos foram classificados como originais.

Já a Figura 3 representa a distribuição dos artigos nas 4 áreas de especialidade da Fonoaudiologia: Linguagem, Motricidade Orofacial, Voz, Saúde Coletiva e Audiologia. Verifica-se que a especialidade Motricidade Orofacial parece despertar maior interesse dos pesquisadores, em virtude de ser esta a que concentra maior número de publicações, considerando os diferentes periódicos. Ao serem considerados os periódicos individualmente, percebe-se que o periódico $C$, além de deter o maior número de publicações, também é o que apresenta maior variedade de artigos relacionados a mais de uma especialidade da Fonoaudiologia, ao contrário dos outros periódicos, cujas publicações concentraram-se apenas na Motricidade Orofacial. Neste gráfico, também percebe-se que as especialidades Voz e Saúde Coletiva não foram contempladas com nenhum estudo sobre EMGs.

$\mathrm{Na}$ Figura 4 é possível visualizar todos os temas pesquisados nestes 10 artigos sobre EMGs. O tema fluência foi o mais recorrente (4), seguido pelo tema mastigação (3). Ainda foram abordados os seguintes temas: paralisia facial, sucção em bebês e distúrbios vestibulares, com 1 artigo cada. Todos os estudos com disfluência foram publicados na revista $\mathrm{C}$ e esta também apresentou maior variedade de temas. 


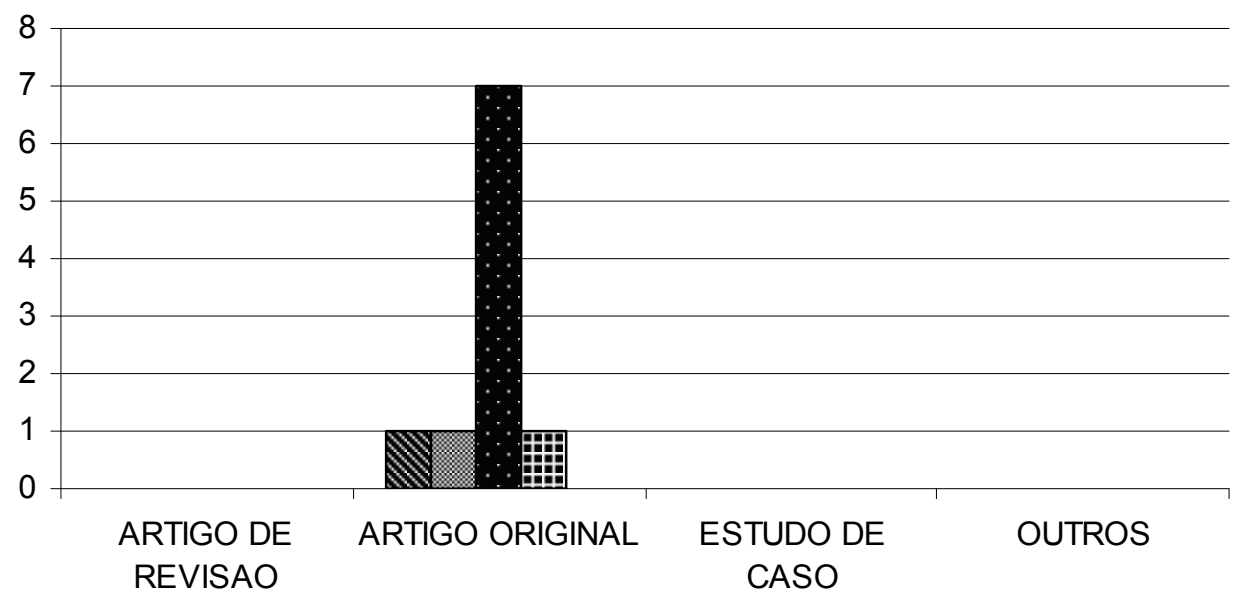

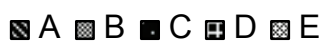

Figura 2 - Distribuição das publicações sobre EMGs nos periódicos nacionais em Fonoaudiologia, segundo o tipo de artigo

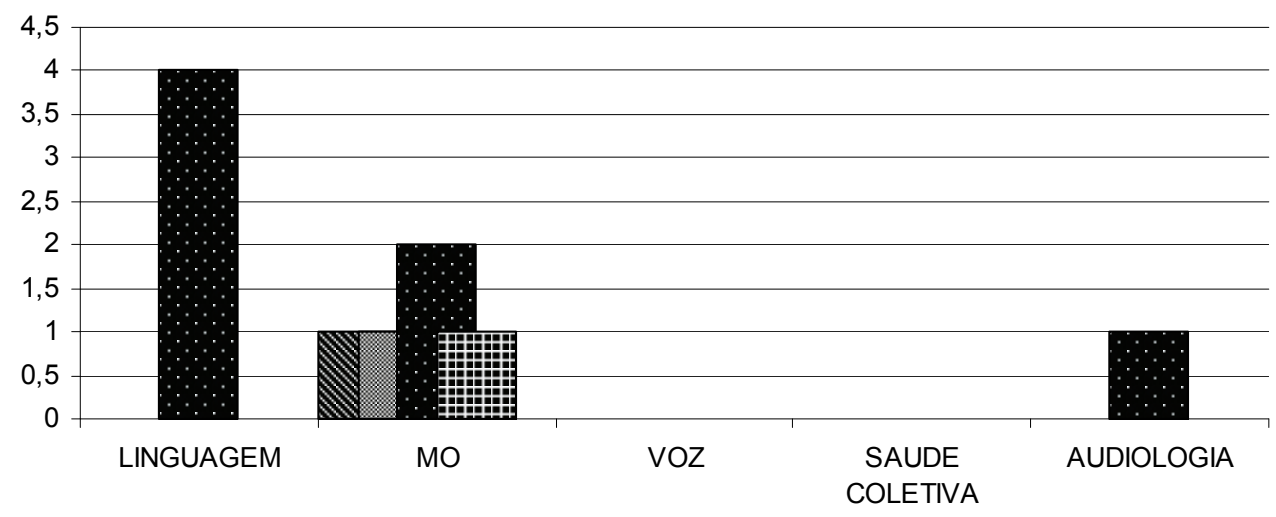

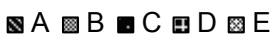

Figura 3 - Distribuição das publicações sobre EMGs nos periódicos nacionais em Fonoaudiologia, segundo a área de especialidade da Fonoaudiologia

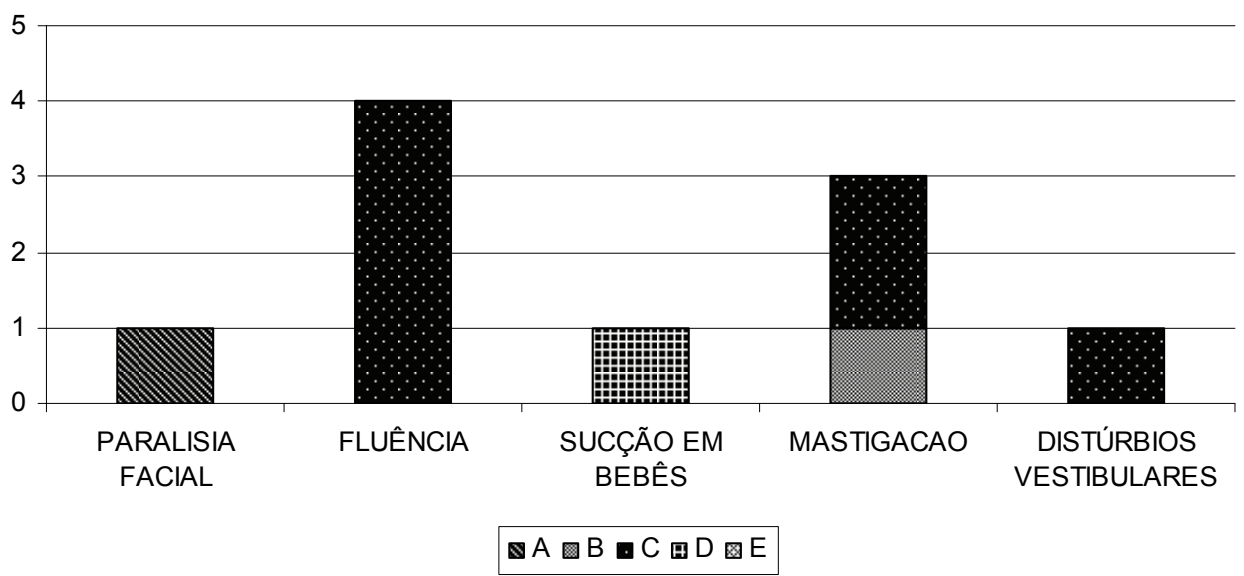

Figura 4 - Distribuição das publicações sobre EMGs nos periódicos nacionais em Fonoaudiologia, segundo tema 
Trabalhos com análise histórica da produção do conhecimento são considerados importantes por estimular novos estudos visando não só o registro histórico do que tem sido pesquisado, mas também auxiliando na definição de ações e campos estratégicos para o implemento de pesquisas ${ }^{22}$.

Os periódicos nacionais em Fonoaudiologia tem buscado aprimorar seus critérios de publicação, buscando uma maior aproximação dos periódicos de alto impacto no que diz respeito a adoção dos critérios na seleção de seus artigos, inclusive se inserindo em novas bases de dados.

Como pôde ser constatado na Tabela 1, em 2006 existiam 5 periódicos no país, porém neste mesmo ano a revista $E$ parou de publicar, assim como a revista B no ano seguinte. Atualmente, o Brasil possui três revistas indexadas especializadas em assuntos relacionados a Fonoaudiologia e todas fazem parte da base de dados LILACS.

Percebe-se pela Tabela 1, que o número total de artigos publicados pelos periódicos entre $2000 \mathrm{e}$ 2008 é expressivo (954 artigos), no entanto, existe escassez de estudos envolvendo a EMGs, totalizando apenas 10 artigos, o que representa cerca de $1 \%$ do total. Essa carência de estudos pode ser justificada por diversos motivos e alguns deles podem ser: inserção recente dos experimentos com EMGs na Fonoaudiologia; difícil acesso de pesquisadores ao método; restrições e limitações do uso da EMGs; estudos realizados por fonoaudiólogos, mas publicados em periódicos nacionais de áreas afins ou internacionais; falta de interesse dos pesquisadores em publicar em periódicos nacionais especializados em Fonoaudiologia; inadequação dos artigos submetidos às normas da revista. Além disso, deve ser considerado o fato de alguns artigos envolvendo EMGs terem sido publicados em alguns dos periódicos estudados antes destes serem indexados.

Em um estudo que procurou caracterizar os periódicos nacionais pelo fator de impacto no período de 1992 a 1998 observou-se que a Fonoaudiologia brasileira ainda precisa desenvolver alguns aspectos em seus processos de difusão científica, sendo necessário que os editores aumentem o acesso e a visibilidade de seus periódicos e os fonoaudiólogos brasileiros citem os trabalhos de seus antecessores e parceiros nacionais. Os autores desse trabalho ainda apontam alguns fatores que podem ter influenciado no resultado encontrado, e que podem ser aplicados aos resultados encontrados no presente estudo, como, por exemplo, a baixa visibilidade da base de dados Lilacs; baixa acessibilidade destes periódicos; pouco interesse dos pesquisadores na leitura de periódicos nacionais; inexistência da participação de bibliotecários experientes e conhecedores das peculiaridades da Fonoaudiologia brasileira na escolha dos descritores de um trabalho científico; combinações dos itens anteriores ${ }^{22}$.

$\mathrm{O}$ fato do periódico $\mathrm{C}$ concentrar o maior número de artigos pode ser justificado por ser esta a única revista que esteve incluída em todo o período considerado nesta análise ou ainda, por ser este um periódico com elevado número de publicações por volume e o que detem melhor Qualis dentre as cinco revistas até o momento em que esta pesquisa foi realizada.

Por outro lado, é possível encontrar periódicos que não publicaram nenhum artigo sobre o assunto, como é o caso da revista $E$, indexada entre 2001 e 2006. Já a revista B, mesmo encerrando sua periodicidade em 2007, publicou apenas 1 artigo relacionado a EMGs em mais de 10 anos de indexação.

Apesar do número ainda inexpressivo, percebese um esboço do aumento no número de pesquisas sendo realizadas por fonoaudiólogos nesta área ao longo dos anos, o que pode ser visualizado na Figura 2. Em 2008, existem mais estudos envolvendo EMGs do que em anos anteriores, refletindo um possível maior interesse dos pesquisadores neste assunto.

Em relação ao tipo de artigo, 100\% foram originais, o que indica não ser este um assunto esgotado e com grande potencial gerador de pesquisas na Fonoaudiologia. O elevado percentual de artigos originais, também foi encontrado em outro estudo que caracterizou as publicações periódicas nacionais em Fonoaudiologia com a produção do conhecimento em Linguagem e Neurociências ${ }^{21}$. Este tipo de artigo é considerado importante para a qualidade de um periódico, pois se destina à divulgação de resultados de pesquisa científica e geralmente são originais e inéditos ${ }^{21,23}$.

Em relação às especialidades da Fonoaudiologia, a Motricidade Orofacial é a que possui maior número de artigos, considerando todos os periódicos analisados. Sendo esta a especialidade que possui uma maior intimidade com o estudo do sistema neuromuscular, tal resultado era esperado. Um número expressivo de estudos na área de Linguagem está relacionado a trabalhos realizados em uma linha de pesquisa que envolve a fluência. Além disso, verifica-se pela Figura 3 que todos os estudos nessa área foram publicados na Revista C, que também privilegiou, porém em menor número, pesquisas em Motricidade Orofacial e Audiologia. Além da Revista $\mathrm{C}$, nenhuma outra revista publicou algum artigo em uma área distinta da Motricidade Orofacial.

A Figura 3 também mostra que as áreas de Voz e Saúde Coletiva não foram prestigiadas, 
não existindo a ocorrência de nenhum estudo nessas duas áreas. $\mathrm{Na}$ área de voz, as pesquisas com EMGs são possíveis ${ }^{24}$, porém em virtude da especificidade dos grupos musculares, a literatura aponta para um maior número de estudos utilizando a eletroneuromiografia ${ }^{25,26}$. A inexistência de publicações nesta especialidade nos periódicos nacionais em Fonoaudiologia indica ser esta uma área a ser explorada e melhor estudada. O grupo de pesquisa Patofisiologia do Sistema Estomatognático, vinculado a Universidade Federal de Pernambuco, ${ }^{27}$ tem a preocupação em explorar esta área. Pesquisas estão sendo realizadas explorando a avaliação eletromiográfica da musculatura extrínseca da laringe de indivíduos disfônicos. Já estudos em Saúde Coletiva, são mais infrequentes, provavelmente pelo caráter essencialmente clínico do método, não invalidando, contudo, que estudos com populações maiores, envolvendo aspectos metodológicos da Epidemiologia possam ser aplicados.

Partindo das especialidades fonoaudiológicas, foram investigados os temas estudados pelos 10 trabalhos encontrados. O resultado está exposto na Figura 4. Foram encontrados cinco temas e como já mencionado, artigos com o tema disfluência foram todos publicados pelo periódico $\mathrm{C}$, sendo este $\mathrm{O}$ tema mais frequente nos 10 artigos.

A mastigação foi o único tema a ser publicado em mais de uma revista (revista $C$ e revista $E$ ). $A$ atividade elétrica dos músculos envolvidos nesta função é largamente estudada em todo o mundo, mas apesar disso, é um tema ainda não esgotado, em virtude da falta de consenso existente entre os trabalhos e pela vasta possibilidade de fenômenos a serem observados envolvendo a mastigação.
A revista $C$ publicou ainda um artigo com o tema distúrbios vestibulares, a revista $\mathrm{A}$ um artigo sobre paralisia facial e a revista $D$, um artigo sobre sucção em bebês.

Assim como o número de artigos encontrados, os temas estudados foram extremamente restritos, dada a enorme variedade de estudos possíveis com a utilização da EMGs, como por exemplo, sua relação com funções como a deglutição e fonação, por exemplo.

Indica ser, portanto, um instrumento ainda pouco prestigiado pelas publicações nacionais em Fonoaudiologia, merecendo mais destaque em virtude do espaço que vem ganhando em pesquisas nacionais e internacionais congregando fonoaudiólogos e profissionais de outras áreas e estabelecendo parâmetros objetivos de avaliação, diagnóstico e acompanhamento terapêutico nas diversas alterações do sistema neuromuscular.

\section{CONCLUSÃO}

Poucos estudos abordam a eletromiografia de superfície nos periódicos nacionais em Fonoaudiologia, com maior concentração nas áreas de motricidade orofacial e linguagem, revelando uma maior necessidade de aprofundar esse assunto e diversificar os temas de interesse fonoaudiológico.

\section{AGRADECIMENTOS}

Os autores agradecem ao Conselho Nacional de Tecnologia e Desenvolvimento Científico (CNPq), que forneceu suporte financeiro através do Edital Universal MCT/CNPq 14/2009 - Faixa B - Processo: 476412/2009-9.

\section{ABSTRACT \\ Theme: surface electromyography in national journals in Speech, Language and Hearing Sciences. Purpose: to characterize the approach of surface electromyography in national journals in Speech, Language and Hearing Sciences. Conclusions: there are few studies about the surface electromyography in national journals in Speech, Language and Hearing Sciences, but most pertain to the Orofacial Motricity and Fluency areas. There seems to be a need to develop this subject and to diversify the topics referring to Speech-Language Pathology interest.}

KEYWORDS: Electromyography; Periodicals; Scientific and Technical Publications 


\section{REFERÊNCIAS}

1. Rodrigues AMM, Bérzin F, Siqueira VCV. Análise eletromiográfica dos músculos masseter e temporal na correção da mordida cruzada posterior. Rev Dent Press Ortodon Ortop Facial. 2006; 11(3):55-62.

2. Biasotto DC, Biasoto-Gonzalez DA, Panhoca I. Correlation between the clinical phonoaudiological assessment and electromyographic activity of the masseter muscle. J Appl Oral Sci. 2005; 13(4):424-30.

3. Rahal A, Pierotti S. Eletromiografia e cefalometria na Fonoaudiologia. In: Ferreira, LP, Befi-Lopes, DM, Limongi, SCO, organizadores. Tratado de fonoaudiologia. São Paulo: Roca; 2004. p. 237-53. 4. Perlman AL, Van Daele DJ. Disfagia: avaliação. In: Bailey BJ, Johnson JT. Coleção otorrinolaringologia: cirurgia de cabeça e pescoço. Vol. 2. Vias aéreas, deglutição, voz. Rio de Janeiro: Revinter; 2010. p.23-32.

5. Vaiman M. Standardization of surface electromyography utilized to evaluate patients with dysphagia. Head Face Med [periódico on line]. 2007; 3(26):7p. [acesso em 23 fev 2009] Disponível em: http://www.head-face-med.com/content/3/1/26.

6. Vaiman M, Eviatar E. Surface electromyography as a screening method for evaluation of dysphagia and odynophagia. Head Face Med [periódico on line]. 2009; 5(9):11p. [acesso em 23 fev 2009] Disponível em: http://www.head-face-med.com/ content/5/1/9.

7. Paiva G, Mazzeto MO. Atlas de placas interoclusais. São Paulo: Santos; 2008.

8. Botelho AL, Brochini APZ, Martins MM, Melchior MO, Silva AMBR, Silva MAMR. Avaliação eletromiográfica de assimetria dos músculos mastigatórios em sujeitos com oclusão normal. RFO. 2008; 13(3):7-12.

9. Soderberg GL, Cook TM. Electromyography in biomechanics. Phys Theraphy. 1984; 64(12):1813-20.

10. Sampaio CRA. Avaliação eletromiográfica dos músculos masseter e temporal anterior após o uso da placa de Hawley modificada em pacientes com DTM [dissertação]: Universidade Federal de Pernambuco; 2003.

11. Rodrigues KA, Rahal A. A influência da tipologia facial na atividade eletromiográfica do músculo masseter durante o apertamento dental em máxima intercuspidação. Rev. CEFAC. 2003; 5:127-30.

12. Felício CM, Couto GA, Ferreira CLP, Mestriner Junior W. Reliability of masticatory efficiency with beads and correlation with the muscle activity. Pró-Fono. 2008; 20(4):225-30.

13. Mangilli LD, Sassi FC, Sernik RA, Tanaka C, Andrade CRF. Avaliação eletromiográfica e ultrassonográfica do músculo masseter em indivíduos normais: estudo piloto. Pró-Fono. 2009; 21(3):261-4.

14. Ferla A, Silva AMT, Corrêa ECR. Atividade eletromiográfica dos músculos temporal anterior e masseter em crianças respiradoras bucais e em respiradoras nasais. Rev Bras Otorrinolaringol. 2008; 74(4):588-95.

15. Nagae M, Bérzin F. Electromyography: applied in phonoaudiolgy clinic. Braz J Oral Sci. 2004; 3(10):506-9

16. Rahal A, Goffi-Gomez MVS. Avaliação eletromiográfica do músculo masseter em pessoas com paralisia facial periférica de longa duração. Rev. CEFAC. 2007; 9(2):207-12.

17. Tartaglia GM, Barozzi S, Marin F, Cesarani A, Ferrario VF. Electromyographic activity of sternocleidomastoid and masticatory muscles in patients with vestibular lesions. J Appl Oral Sci. 2008; 16(6):391-6.

18. Berretin-Félix G, Nary Filho H, Padovani CR, Trindade Junior AS, Machado WM. Electromyograpic evaluation of mastication and swallowing in eldery individuals with mandibular fixed implant-supported prosthesis. J Appl Oral Sci. 2008; 16(2):116-21.

19. Berberian AP, Ferreira LP, Corteletti LCBJ, Azevedo JBM, Marques JM. A produção do conhecimento em distúrbios da comunicação: análise de periódicos (2005-2005). Rev Soc Bras Fonoaudiol. 2009; 14(2):153-9.

20. Brasil. Ministério da Educação. Coordenadoria de Aperfeiçoamento de Pessoal de Nível Superior (Capes). Classificação de periódicos, anais, revistas e jornais. [homepage na internet] Brasília. [acesso em 18 nov 2009]. Disponível em: http:// qualis.capes.gov.br.

21. Vasconcelos SV, Pessoa ACRG, Farias APS. Caracterização das publicações periódicas em fonoaudiologia e neurociências: estudo sobre os tipos e temas de artigos e visibilidade na área de linguagem. Rev. CEFAC. 2009; 11(1):50-8.

22. Campanatti-Ostiz H, Andrade CRF. Periódicos nacionais em Fonoaudiologia: caracterização de indicador de impacto. Pró-Fono. 2006; 18(1):99-110.

23. Krzyzanowski RF, Ferreira MCG. Periódicos científicos: critérios de qualidade. Rev Odontol Bras. 2003; 17(1):43-8.

24. Jacoby P, Rabine E, Rohmert G, Rohmert W, Timm C, Zipp P. Surface electromyography for the study of voice function. Folia Phoniatr (Basel). 1984; 36(5):241-9.

25. Ramalho JRO, Corso RJ, Ferreira GD, Pinto HCF, Pinto JA, Catai AL. Eletromiografia de laringe. Rev. Bras. Otorrinolaringol. 2001; 67(4pt.1):536-42. 
26. Correa G, Otarola F, Del Lago J. Electromiografía laríngea. Rev. Otorrinolaringol. Cir. Cabeza Cuello. 2000; 60(2):91-8.

27. Grupo de Pesquisa Patofisiologia do sistema estomatognático. Diretório de Grupos de pes- quisa CNPq. [homepage na internet] Brasília. [acesso em 18 nov 2009]. Disponível em: http:// dgp.cnpq.br/buscaoperacional/detalhegrupo. jsp?grupo=0021407EOZ9GQK

RECEBIDO EM: 22/11/2009

ACEITO EM: 14/01/2010

Endereço para correspondência:

Leandro de Araújo Pernambuco

Rua Waldemar Lima, 336

Olinda - PE

CEP:53110-630

E-mail: leandroape @globo.com 